\title{
Sensibilidad de aislamientos de Phytophthora infestans procedentes de Solanum tuberosum a tres fungicidas sistémicos
}

\section{Solanum tuberosum isolate sensitivity to Phytophthora infestans when using three systemic fungicides}
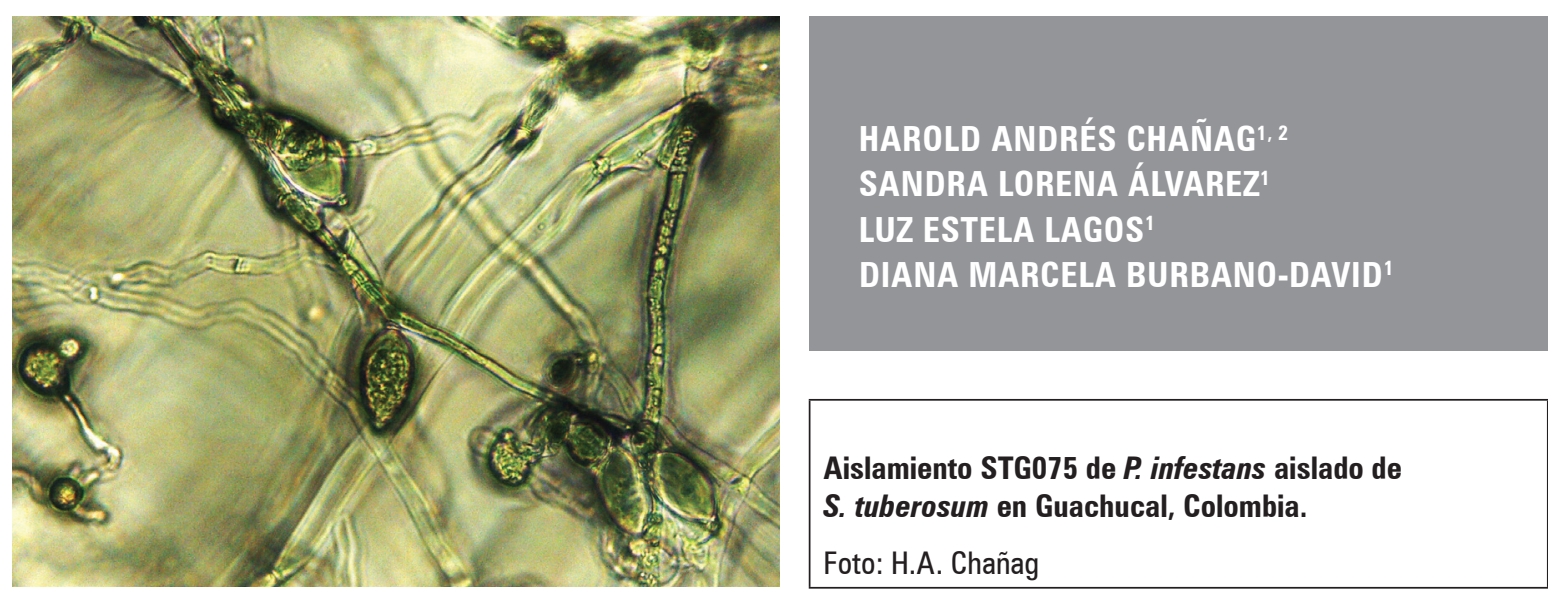

\section{RESUMEN}

Phytophthora infestans, es el agente causal de una de las enfermedades más limitantes de cultivos de papa (Solanum tuberosum) en el departamento de Nariño causando que los agricultores utilicen de forma intensiva diferentes fungicidas, llegando a representar el 30\% de los costos de producción, siendo un grave problema ambiental y de salud pública. El propósito de esta investigación es apoyar los programas de manejo integrado de enfermedades a partir de la generación de una línea base de sensibilidad de 20 aislamientos de $P$. infestans del departamento de Nariño frente a tres fungicidas sistémicos comerciales compuestos por Metalaxil $8 \%+$ Mancozeb 64\%, Cimoxanil 6\% + Propineb 70\% y Fluopicolide 6\% + Propineb 66,7\%. Este estudio se llevó a cabo siguiendo la metodología in vitro utilizando cinco dosis basadas en una escala exponencial. Los resultados mostraron un comportamiento heterogéneo donde algunos aislamientos presentaron resistencia al fungicida compuesto por Metalaxil + Mancozeb, alcanzando valores promedio de EC50 de 6,67 $\mu \mathrm{g} \mathrm{mL}^{-1}$, mientras que para los fungicidas compuestos por Cimoxanil + Propineb y Fluopicolide + Propineb se presentaron niveles de sensibilidad intermedia y sensibles con valores de EC50 promedio de $1,57 \mu \mathrm{g} \mathrm{mL}^{-1}$ y 2,61 $\mu \mathrm{g} \mathrm{mL}^{-1}$. Por otra parte, la tasa de esporulación se redujo en su totalidad para los tres fungicidas cuando se evaluaron las dosis de $100,1.000,10.000 \mu \mathrm{g} \mathrm{mL}^{-1}$.

\footnotetext{
Palabras clave adicionales: resistencia a los fungicidas; Peronosporales; cimoxanil; fluopicolide; metalaxil; papa.

Universidad de Nariño, Grupo de Investigación Genética y Evolución de Organismos Tropicales, San Juan de Pasto (Colombia). ORCID Chañag, H.A.: 0000-0002-2749-0367; ORCID Álvarez, S.L.: 0000-0002-9464-5429; ORCID Lagos, L.E.: 0000-0002-5152-3413; Burbano-David, D.M.: 0000-0003-0109-3088

2 Autor para correspondencia. harold.a963@hotmail.com
} 


\section{ABSTRACT}

Phytophthora infestans is the causative agent of one of the most limiting diseases in potato crops (Solanum tuberosum) in the Department of Nariño, causing farmers to intensively use different fungicides, which reach $30 \%$ of production costs and are a serious environmental and public health problem. The purpose of this research was to support integrated disease management programs based on the generation of a baseline sensitivity of $20 P$. infestans isolates from the Department of Nariño to three commercial fungicides composed of Metalaxyl 8\% + Mancozeb 64\% , Cimoxanil 6\% + Propineb 70\% and Fluopicolide 6\% + Propineb 66.7\%. This study was carried out using the in vitro methodology and five doses on an exponential scale. The results showed a heterogeneous behavior where some isolates showed resistance to the fungicide composed of Metalaxil + Mancozeb, reaching average EC50 values of $6.67 \mu \mathrm{g} \mathrm{mL}^{-1}$; while for the fungicides composed of Cimoxanil + Propineb and Fluopicolide + Propineb, there were intermediate and sensitive sensitivity levels with average EC50 values of $1.57 \mu \mathrm{g} \mathrm{mL}^{-1}$ and $2.61 \mu \mathrm{g} \mathrm{mL}$. On the other hand, the sporulation rate was reduced in its entirety with the three fungicides for the evaluated doses of 100; 1,$000 ; 10,000 \mu \mathrm{g} \mathrm{mL}^{-1}$.

Additional key words: resistance to fungicides; Peronosporal; cymoxanil; fluopicolide; metalaxil; potato.

Fecha de recepción: 13-03-2018 Aprobado para publicación: 30-11-2018

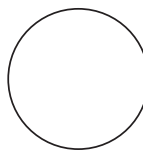

INTRODUCCIÓN

La "gota" o "tizón tardío" es uno de los problemas más limitantes del cultivo de papa causado por el patógeno Phytophthora infestans, esta enfermedad es capaz de afectar el follaje, tallos y el tubérculo en cualquier momento de desarrollo de la planta incluso en el almacenamiento (Marín y Mira, 1998) de no realizarse un control adecuado y preciso esta enfermedad puede llegar a causar el 100\% de severidad (Montes et al., 2012). Esta enfermedad cobra importancia en el país puesto que para el 2017 se cosecharon 162.879 ha de papa con una producción de 3’706.563 t, siendo los departamentos de Cundinamarca, Boyacá y Nariño los principales productores (DANE - ENA, 2018), donde se siembran materiales altamente susceptibles como las variedades Diacol Capiro, Parda Pastusa, ICA Nevada y Tuquerreña que, además, los municipios productores presentan condiciones climáticas favorables para el desarrollo de esta enfermedad.

La alternativa más eficiente para el control de esta problemática se basa principalmente en el uso intensivo de fungicidas con moléculas protectantes y sistémicas (Granada et al., 2002) que representa un costo que oscila entre 10 y $30 \%$ del valor total de la producción de los cultivos de papa en el país (Jaramillo, 2004). Siendo los productos con moléculas sistémicas los más eficientes que presentan diferentes modos de acción como, por ejemplo, metalaxil afecta la síntesis de ARN ribosomal y por ende la síntesis de proteínas reduciendo el crecimiento del micelio (Nuninger et al., 1995); Cimoxanil por su parte posee un modo de acción multisitio y translaminar que incluye la inhibición de la síntesis de ADN, ARN, aminoácidos y lípidos, además afecta la respiración celular y la permeabilidad de la membrana celular (Erwin y Ribeiro, 1996; Garcia et al., 2008) y finalmente, Fluopicolide, ingrediente activo relativamente nuevo que se clasifica como piridinilmetil-benzamida que interrumpe la división celular y la mitosis actuando sobre las proteínas como la espectrina encargada de la estabilidad del citoesqueleto del patógeno (Briggs et al., 2006; FRAC, 2018).

El uso intensivo (hasta 18 aspersiones durante el ciclo del cultivo) de fungicidas sistémicos a lo largo del tiempo generan resistencia lo que se define como la disminución temporal de la sensibilidad a un determinado fungicida, resultado de la adaptación del patógeno. Así, por ejemplo, en el año 2001 se determinó que cerca del $75 \%$ de los aislamientos colectados en el departamento de Antioquia resultaron insensible a Metalaxil (Jaramillo, 2004), mientras que los resultados de sensibilidad a Cimoxanil han resultado variables (Ochoa, 2004).

Teniendo en cuenta la problemática el objetivo de la presente investigación se centró en determinar el nivel de sensibilidad de aislamientos de P. infestans a tres fungicidas sistémicos, con el fin de establecer acciones de monitoreo que apoyen a la toma de decisiones 
relacionadas con el uso de fungicidas de síntesis química en el cultivo de la papa en Colombia, puesto que la característica de resistencia al no ser heredable puede ser revertida por cambios en las estrategias de control químico (Pérez y Forbes, 2008).

\section{MATERIALES Y MÉTODOS}

\section{Material evaluado}

Las pruebas de sensibilidad se realizaron con 20 aislamientos de $P$. infestans obtenidos de Solanum tuberosum y $S$. phureja en el Departamento de Nariño y hacen parte del cepario del grupo de investigación de Genética y Evolución de Organismos Tropicales de la Universidad de Nariño (Tab. 1).

Tabla 1. Información aislamiento aislamientos de Phytophthora infestans.

\begin{tabular}{|c|c|c|}
\hline $\begin{array}{c}\text { Código } \\
\text { aislamiento }\end{array}$ & Localidad & Hospedero \\
\hline SPP031 & Pasto & S. phureja \\
\hline SPT055 & Túquerres & S. phureja \\
\hline SPT056 & Túquerres & S. phureja \\
\hline STA083 & Aldana & S. tuberosum \\
\hline STG075 & Guachucal & S. tuberosum \\
\hline STG105 & Pasto & S. tuberosum \\
\hline ST1155 & Ipiales & S. tuberosum \\
\hline ST1169 & Ipiales & S. tuberosum \\
\hline STP062 & Pasto & S. tuberosum \\
\hline STP063 & Pasto & S. tuberosum \\
\hline STP067 & Pasto & S. tuberosum \\
\hline STP113 & Pasto & S. tuberosum \\
\hline STS035 & Sapuyes & S. tuberosum \\
\hline STS037 & Sapuyes & S. tuberosum \\
\hline STT144 & Túquerres & S. tuberosum \\
\hline STT146 & Túquerres & S. tuberosum \\
\hline STT159 & Túquerres & S. tuberosum \\
\hline STT161 & Túquerres & S. tuberosum \\
\hline STT162 & Túquerres & S. tuberosum \\
\hline
\end{tabular}

\section{Determinación de sensibilidad in vitro de los aislamientos}

Para la determinación del efecto de los fungicidas sobre el patógeno se utilizaron los productos comerciales compuestos por Metalaxil 8\% + Mancozeb 64\%, Cimoxanil 6\% + Propineb $70 \%$ y Fluopicolide $6 \%+$ Propineb $66,7 \%$, con el fin de asegurar la reproducibilidad del ensayo se hicieron tres replicas $y$ tres repeticiones en el tiempo de cada tratamiento y un testigo absoluto sin fungicida. Las dosis evaluadas se calcularon en base a una escala exponencial, inicialmente se prepararon las soluciones stock a una concentración de $100.000 \mu \mathrm{g} \mathrm{mL}^{-1}$ a partir de las cuales se prepararon las diferentes concentraciones que fueron adicionadas en el medio de cultivo agar tomate - arveja. El inoculo de $5 \mathrm{~mm}$ de diámetro se tomó de la zona de crecimiento activo de cultivos de $10 \mathrm{~d}$ y fue ubicado en el centro de cada caja petri con agar Tomate Arveja previamente suplementada con fungicida. Una vez efectuada la inoculación, las cajas petri se sellaron herméticamente y se incubaron a temperatura ambiente y oscuridad durante $10 \mathrm{~d}$, desde el primer día se registró fotográficamente el crecimiento micelial y con los resultados de crecimiento final se calculó el porcentaje de inhibición.

Al décimo día se tomó el micelio formado en cada caja petri y se depositó en un volumen de 1,5 $\mathrm{mL}$ de agua destilada estéril, posteriormente se sometió a vortex por $30 \mathrm{~s}$ y se tomaron $100 \mu \mathrm{L}$ de esta solución para realizar el conteo de esporangios en cámara de Neubauer y se expresó como numero de esporangios sobre área de crecimiento final para cada uno de los aislamientos por tratamiento.

\section{Determinación $\mathrm{EC}_{50}$}

Se realizó un análisis Probit para encontrar la $\mathrm{EC}_{50}$ (concentración que reduce el crecimiento radial en un $50 \%$ respecto al control) con los valores de inhibición y concentración para cada uno de los aislamientos basado en un análisis de regresión lineal. Teniendo en cuenta los valores de $\mathrm{EC}_{50}$ calculados y de acuerdo con la clasificación de sensibilidad establecida por Wang et al. (2002).

\section{Análisis de datos}

Para determinar la existencia de diferencias significativas en esta investigación se realizaron tablas de contingencia con el programa Microsoft office Excel y se aplicó la prueba de Kruskal Wallis para comparar las concentraciones en el programa Minitab ${ }^{\circledR}$ 17.1.0 (C) 2013 Minitab Inc). 


\section{RESULTADOS}

\section{Metalaxil 8\% + Mancozeb 64\%}

Estos fungicidas redujeron el crecimiento del patógeno entre el 29 y $69 \%$ con las dosis 1 y $10 \mu \mathrm{g} \mathrm{mL}^{-1}$, respectivamente, alcanzando el $100 \%$ de reducción del crecimiento cuando se utilizaron las dosis $100 \mu \mathrm{g}$ $\mathrm{mL}^{-1}, 1.000 \mu \mathrm{g} \mathrm{mL}^{-1}$ y $10.000 \mu \mathrm{g} \mathrm{mL}^{-1}$ las cuales fueron letales para el inóculo. La prueba de Kruskal Wallis mostró que existen diferencias estadísticamente significativas $(P \leq 0,05)$ entre el control y los tratamientos (Tab. 2). Por otra parte, la acción antiesporulante de este fungicida se hizo efectiva a partir del tratamiento correspondiente a la dosis de $100 \mu \mathrm{g} \mathrm{mL}^{-1}$ hasta la dosis de $10.000 \mu \mathrm{g} \mathrm{mL}^{-1}$ inhibiendo el $100 \%$ la producción de esporangios (Tab. 3).

A partir de los resultados de inhibición se obtuvo el valor de la dosis media efectiva $\left(\mathrm{EC}_{50}\right)$, encontrando que estos variaron entre $3,26 \mu \mathrm{g} \mathrm{mL}^{-1}$ en el aislamiento STA084 hasta 12,21 $\mu \mathrm{g} \mathrm{mL}^{-1}$ en el aislamiento STP046 con un promedio de $6,67 \mu \mathrm{g} \mathrm{mL}^{-1}$ (Fig. 1) catalogando el $20 \%$ de los aislamientos resistentes y el $80 \%$ con sensibilidad intermedia.

\section{Cimoxanil 6\% + Propineb 70\%}

La concentración de $1 \mu \mathrm{g} \mathrm{mL} \mathrm{m}^{-1}$ causó una reducción promedio del $25 \%$ del crecimiento del patógeno con respecto al control y algunos aislamientos presentaron una alta sensibilidad a este producto, puesto que con la dosis mínima sus crecimientos fueron inhibidos en un 45\% (aislamiento STI168) y 40\% (aislamiento STT162). Cuando se evaluó la dosis de 10 $\mu \mathrm{g} \mathrm{mL}{ }^{-1}$, los porcentajes de inhibición presentaron un promedio del $89 \%$, mientras que en las dosis superiores a esta (100, 1.000 y $\left.10.000 \mu \mathrm{g} \mathrm{mL}^{-1}\right)$ el porcentaje de inhibición fue 100\% letal para el inóculo. Se encontraron diferencias significativas $(P \leq 0,05)$ entre el control y las concentraciones evaluadas $(1,10,100$, 1.000, $\left.10.000 \mathrm{ug} \mathrm{mL}^{-1}\right)$. Asimismo, hubo diferencias estadísticas $(P \leq 0,05)$ entre los tratamientos evaluados siendo las concentraciones de 1 y $10 \mu \mathrm{g} \mathrm{mL}^{-1}$ diferentes a las demás concentraciones (Tab. 4). La acción antiesporulante de este fungicida se evidenció a partir de la dosis $100 \mu \mathrm{g} \mathrm{mL}^{-1}$. El análisis estadístico mostró que existen diferencias estadísticas entre los tratamientos evaluados, siendo las dosis 1 y $10 \mu \mathrm{g} \mathrm{mL}^{-1}$ diferentes $(P \leq 0,05)$ a las dosis 100 hasta $10.000 \mu \mathrm{g}$ $\mathrm{mL}^{-1}$, concentraciones en las cuales se inhibió el 100\% la esporulación en todos los aislamientos (Tab. 5).

Tabla 2. Prueba de Kruskal Wallis (Bonferroni corrected) para porcentaje de inhibición entre concentraciones del fungicida Metalaxil 8\% + Mancozeb 64\%.

\begin{tabular}{|c|c|c|c|c|c|c|}
\hline Dosis $\left(\mu \mathrm{g} \mathrm{mL}^{-1}\right)$ & Control & 1 & 10 & 100 & 1.000 & 10.000 \\
\hline Control & 1 & & & & & \\
\hline 1 & 0,02863 & 1 & & & & \\
\hline 10 & 0,00044 & 0,18492 & 1 & & & \\
\hline 100 & 0 & 0 & 0,00002 & 1 & & \\
\hline 1.000 & 0 & 0 & 0,00002 & 1 & 1 & \\
\hline 10.000 & 0 & 0 & 0,00002 & 1 & 1 & 1 \\
\hline
\end{tabular}

Tabla 3. Prueba de Kruskal Wallis (Bonferroni corrected) para tasa de esporulación entre concentraciones del fungicida Metalaxil 8\% + Mancozeb $64 \%$.

\begin{tabular}{|c|c|c|c|c|c|c|}
\hline Dosis $\left(\mu \mathrm{gL}^{-1}\right)$ & Control & 1 & 10 & 100 & 1.000 & 10.000 \\
\hline Control & 1 & & & & & \\
\hline 1 & 0,52906 & 1 & & & & \\
\hline 10 & 0,51616 & 0,98418 & 1 & & & \\
\hline 100 & 0 & 0 & 0 & 1 & & 1 \\
\hline 1.000 & 0 & 0 & 0 & 1 & 1 & 1 \\
\hline 10.000 & 0 & 0 & 0 & 1 & 1 & 1 \\
\hline
\end{tabular}




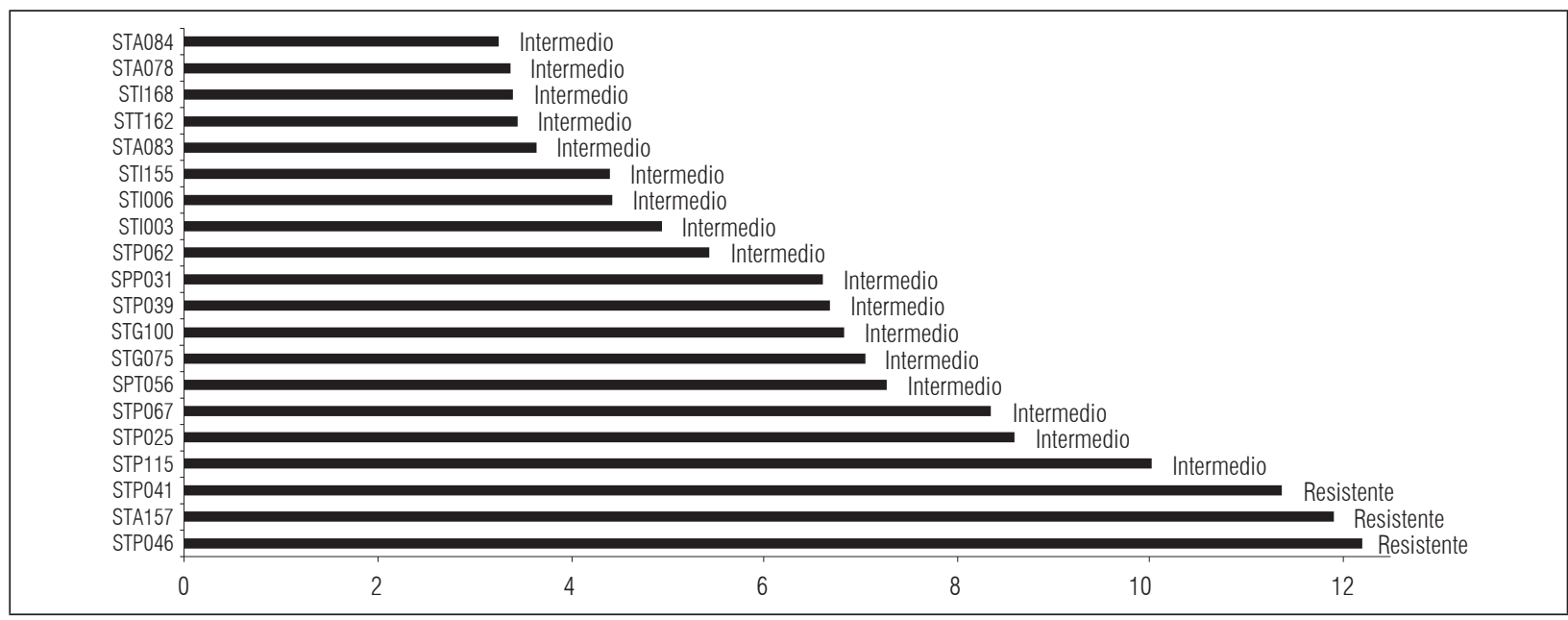

Figura 1. Dosis media efectiva $\left(\mathrm{EC}_{50}\right)$ para aislamientos de Phytophthora infestans frente a Metalaxil + Mancozeb.

Tabla 4. Prueba de Kruskal Wallis (Bonferroni corrected) para porcentaje de inhibición entre concentraciones del fungicida Cimoxanil 6\% + Propineb 70\%.

\begin{tabular}{|c|c|c|c|c|c|c|}
\hline Dosis $\left(\mu \mathrm{g} \mathrm{mL}^{-1}\right)$ & Control & 1 & 10 & 100 & 1.000 & 10.000 \\
\hline Control & 1 & & & & & \\
\hline 1 & 0,05047 & 1 & & & & \\
\hline 10 & 0,00005 & 0,03549 & 1 & & & \\
\hline 100 & 0 & 0 & 0,002 & 1 & & \\
\hline 1.000 & 0 & 0 & 0,002 & 1 & 1 & \\
\hline 10.000 & 0 & 0 & 0,002 & 1 & 1 & 1 \\
\hline
\end{tabular}

Tabla 5. Prueba de Kruskal Wallis (Bonferroni corrected) para tasa de esporulación entre concentraciones del fungicida Cimoxanil 6\% + Propineb $70 \%$.

\begin{tabular}{|c|c|c|c|c|c|c|}
\hline Dosis $\left(\mu \mathrm{g} \mathrm{mL}^{-1}\right)$ & Control & 1 & 10 & 100 & 1.000 & 10.000 \\
\hline Control & 1 & & & & & \\
\hline 1 & 0,87894 & 1 & & & & \\
\hline 10 & 0,95142 & 0,83114 & 1 & & & \\
\hline 100 & 0 & 0 & 0 & 1 & & \\
\hline 1.000 & 0 & 0 & 0 & 1 & 1 & \\
\hline 10.000 & 0 & 0 & 0 & 1 & 1 & 1 \\
\hline
\end{tabular}

Con respecto a los valores de $\mathrm{EC}_{50}$ para este fungicida se encontró que los valores oscilaron entre 0,02853 $\mu \mathrm{g} \mathrm{mL} \mathrm{mL}^{-1}$ (aislamiento STI168) y $6,8116 \mu \mathrm{g} \mathrm{mL}^{-1}$ (aislamiento STP046) con un promedio de 1,575 (Fig. 1). Estos valores permitieron clasificar los asilamientos siguiendo la escala propuesta por Wang et al. (2002) encontrando que el $85 \%$ presentaron sensibilidad intermedia, el 15\% sensibilidad y ninguno resistencia a este fungicida.

\section{Fluopicolide 6\% + Propineb 66,7\%}

Los resultados mostraron que la combinación fúngica reduce el crecimiento del patógeno respecto al control en un $28,4 \%$ en la dosis $1 \mu \mathrm{g} \mathrm{mL}^{-1}$, algunos aislamientos presentaron valores bajos de inhibición con un 3,59\% (aislamiento STP067), 5,06\% (aislamiento STP056) y 6,91\% (aislamiento STG075), lo que puede indicar que estos aislamientos pueden llegar a 
presentar un comportamiento resistente a futuro. Al evaluar la dosis $10 \mu \mathrm{g} \mathrm{mL}^{-1}$, los porcentajes de inhibición presentaron un promedio de $79,26 \%$, mientras que las dosis mayores, entre 100 a $10.000 \mu \mathrm{g} \mathrm{mL}^{-1}$, producen una inhibición del 100\% (Tab. 6).

La actividad antiesporulante se evidenció a partir de la dosis $100 \mu \mathrm{g} \mathrm{mL} \mathrm{m}^{-1}$. El análisis estadístico mostró existencia de diferencias significativas entre las dosis 100, 1.000 y $10.000 \mu \mathrm{g} \mathrm{mL}^{-1}$ frente al control $(P \leq 0,05)$, en los cuales no se registró conteo de esporangios debido a que estas dosis resultaron letales al inóculo (Tab. 7).

\section{DISCUSIÓN}

\section{Metalaxil 8\% + Mancozeb 64\%}

Los resultados encontrados para la inhibición de crecimiento y esporulación corresponden a la característica del metalaxil como molécula altamente sistémica que afecta la síntesis de ARN ribosomal y la síntesis de las proteínas causando la reducción del crecimiento micelial y el desarrollo de esporangios del patógeno (Grunwald et al., 2006).
Los resultados de la dosis media efectiva $\left(\mathrm{EC}_{50}\right)$ para este fungicida concuerdan con los reportados por Lagos (2002) al encontrar después de evaluar 63 aislamientos procedentes del Departamento de Nariño un $24 \%$ de reacción resistente. Dentro de las razones que expliquen la ausencia de poblaciones resistentes a metalaxil en este estudio, sean los cambios presentados en el ingrediente activo, ya que desde el año 2002 se ha distribuido en el mercado variantes dos veces más efectiva que el genérico conocido como Metalaxil - M. De igual manera, el uso intensivo de esta molécula para el control del tizón tardío en la zonas productoras de papa se ha visto reducida en virtud a la aparición de productos comerciales compuestos por nuevas mezclas que contienen fungicidas de tipo preventivo y curativo como Cimoxanil, Dimetomorf, Propamocarb o Estrobulinas (Santana et al., 2013) y que resultan eficientes en el control del patógeno generando una sustitución gradual del metalaxil. Lo que indica que en general, las zonas productoras de papa en el Departamento de Nariño no generan una fuerte presión de selección y que aún se puede considerar el uso de productos a base de Metalaxil en programas de manejo que controlen este patógeno.

Tabla 6. Prueba de Kruskal Wallis (Bonferroni corrected) para porcentaje de inhibición entre concentraciones del fungicida Fluopicolide 6\% + Propineb 66,7\%.

\begin{tabular}{|c|c|c|c|c|c|c|}
\hline Dosis $\left(\mu \mathrm{g} \mathrm{mL}^{-1}\right)$ & Control & $\mathbf{1}$ & $\mathbf{1 0}$ & $\mathbf{1 0 0}$ & $\mathbf{1 . 0 0 0}$ & $\mathbf{1 0 . 0 0 0}$ \\
\hline Control & 1 & & & & & \\
\hline 1 & 0,05074 & 1 & & & & \\
\hline 10 & 0,0001 & 0,05249 & 1 & & & \\
\hline 100 & 0 & 0 & 0,0001 & 1 & & \\
\hline 1.000 & 0 & 0 & 0,0001 & 1 & 1 & \\
\hline 10.000 & 0 & 0 & 0,0001 & 1 & 1 & 1 \\
\hline
\end{tabular}

Tabla 7. Prueba de Kruskal Wallis (Bonferroni corrected) para tasa de esporulación entre concentraciones del fungicida Fluopicolide 6\% + Propineb 66,7\%.

\begin{tabular}{|c|c|c|c|c|c|c|}
\hline Dosis $\left(\mu \mathrm{gL}^{-1}\right)$ & Control & 1 & 10 & 100 & 1.000 & 10.000 \\
\hline Control & 1 & & & & & \\
\hline 1 & 0,65718 & 1 & & & & \\
\hline 10 & 0,11186 & 0,25176 & 1 & & & \\
\hline 100 & 0 & 0 & 0 & 1 & & 1 \\
\hline 1.000 & 0 & 0 & 0 & 1 & 1 & 1 \\
\hline 10.000 & 0 & 0 & 0 & 1 & 1 & \\
\hline
\end{tabular}


Los bajos niveles de sensibilidad del $20 \%$ de los aislamientos de $P$. infestans evaluados frente al fungicida compuesto por Metalaxil + Mancozeb encontrados

Tabla 8. Clasificación de aislamientos de $P$. infestans según los valores de dosis media efectiva $\left(\mathrm{EC}_{50}\right)$ del fungicida Metalaxil 8\% + Mancozeb 64\%.

\begin{tabular}{|c|c|c|}
\hline Cepas & EC $_{50}$ & Clasificación \\
\hline STP046 & 12,21 & Resistente \\
\hline STA157 & 11,91 & Resistente \\
\hline STP041 & 11,39 & Resistente \\
\hline STP115 & 10,03 & Resistente \\
\hline STP025 & 8,62 & Intermedio \\
\hline STP067 & 8,38 & Intermedio \\
\hline SPT056 & 7,29 & Intermedio \\
\hline STG075 & 7,05 & Intermedio \\
\hline STG100 & 6,84 & Intermedio \\
\hline STP039 & 6,70 & Intermedio \\
\hline SPP031 & 6,63 & Intermedio \\
\hline STP062 & 5,46 & Intermedio \\
\hline ST1003 & 4,95 & Intermedio \\
\hline STI006 & 4,44 & Intermedio \\
\hline ST1155 & 4,41 & Intermedio \\
\hline STA083 & 3,65 & Intermedio \\
\hline STT162 & 3,45 & Intermedio \\
\hline ST1168 & 3,42 & Intermedio \\
\hline STA078 & 3,38 & Intermedio \\
\hline STA084 & 3,26 & Intermedio \\
\hline
\end{tabular}

en este estudio conducen a redoblar los esfuerzos por implementar estrategias anti-resistencia en el Departamento de Nariño, según recomendadas de FRAC (2018) para el manejo de estos fungicidas y descritas por García et al. (2008) en los siguientes aspectos: implementar prácticas culturales de control, utilizar mezclas que incluyan fungicidas de tipo fenilaminas con productos protectantes multisitio en proporciones superiores al $75 \%$ y limitar el uso curativo o erradicarte de dichos fungicidas limitando el uso entre 2 a 4 aplicaciones por ciclo de cultivo en intervalos mínimos de $14 \mathrm{~d}$.

\section{Cimoxanil 6\% + Propineb 70\%}

La eficiencia encontrada en la inhibición de producción de esporangios y crecimiento del patógeno, se deba a que este fungicida compuesto por Cimoxanil, un ingrediente activo que posee un modo de acción multisitio, incluye la inhibición de la síntesis de ADN, ARN, aminoácidos y lípidos, además de afectar la respiración celular y la permeabilidad de la membrana celular inhibiendo el crecimiento de micelio del oomycete durante el periodo de incubación (Escudero, 2005).

Con respecto a los valores de $\mathrm{EC}_{50}$ se encontró que los valores oscilaron entre $0.02853 \mu \mathrm{g} \mathrm{mL}^{-1}$ (aislamiento STI168) y $6,8116 \mu \mathrm{g} \mathrm{mL}^{-1}$ (aislamiento STP046), con un promedio de 1,575 (Fig. 2). Estos valores permitieron clasificar los asilamientos siguiendo la escala propuesta por Wang et al. (2002) encontrando que el 85\% presentaron sensibilidad intermedia, el 15\% sensibilidad y ninguno de los aislamientos mostró resistencia.

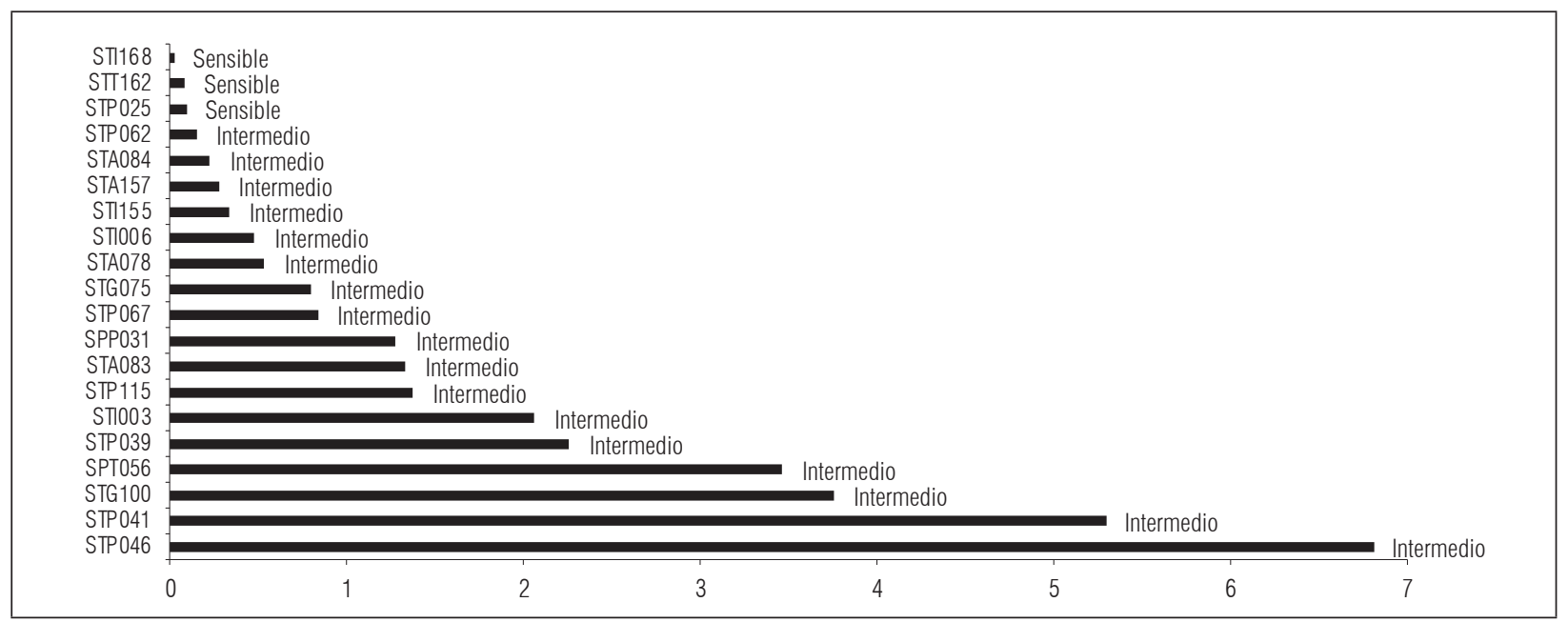

Figura 2. Dosis media efectiva $\left(\mathrm{EC}_{50}\right.$ ) para aislamientos de Phytophthora infestans frente a Cimoxanil + Propineb. 
Al comparar los valores encontrados de $\mathrm{EC}_{50}$ con otros estudios realizados en el país, se encuentra que es frecuente este comportamiento de sensibilidad en aislamientos de P. infestans frente a Cimoxanil. Estudios realizados por Lagos (2002) mostraron que de 64 aislamientos de $P$. infestans evaluados y procedentes de Nariño, el $83 \%$ resultó sensible al ingrediente activo Cimoxanil y ninguno alcanzó el nivel de clasificación de resistente. De igual forma, Ochoa (2004) al evaluar 50 aislamientos obtenidos de diferentes regiones de Colombia, encontró que ninguno tuvo la capacidad de crecer en medios de cultivos suplementados con una concentración del ingrediente activo Cimoxanil al $0,8 \mu \mathrm{g} \mathrm{mL}^{-1}$, estos resultados demuestran que en las poblaciones evaluadas no se reportó aislamientos resistentes a la molécula. En cuanto a evaluaciones con el producto comercial García et al. (2008) reporta que para poblaciones de $P$. infestans aisladas de los departamentos de Antioquia, Boyacá y Cundinamarca frente a Curzate M-8 (DuPont ${ }^{\mathrm{TM}}$ ), se observan altos niveles de sensibilidad con valores promedios de $\mathrm{EC}_{50}$ de 0,20 $\mu \mathrm{g} \mathrm{mL} L^{-1}$.

Tabla 9. Clasificación de aislamientos de $P$. infestans según los valores de dosis media efectiva $\left(\mathrm{EC}_{50}\right)$ del fungicida Cimoxanil 6\% + Propineb 70\%.

\begin{tabular}{|c|c|c|}
\hline Aislamiento & EC $_{50}$ & Clasificación \\
\hline STP046 & 6.81167519 & Intermedio \\
\hline STP041 & 5.29215813 & Intermedio \\
\hline STG100 & 3.75804642 & Intermedio \\
\hline SPT056 & 3.46753449 & Intermedio \\
\hline STP039 & 2.26203998 & Intermedio \\
\hline STI003 & 2.06494207 & Intermedio \\
\hline STP115 & 1.37711758 & Intermedio \\
\hline STA083 & 1.33328641 & Intermedio \\
\hline SPP031 & 1.28169424 & Intermedio \\
\hline STP067 & 0.83474941 & Intermedio \\
\hline STG075 & 0.79677689 & Intermedio \\
\hline STA078 & 0.53733513 & Intermedio \\
\hline STI006 & 0.48214208 & Intermedio \\
\hline STI155 & 0.33825317 & Intermedio \\
\hline STA157 & 0.27725433 & Intermedio \\
\hline STA084 & 0.23039431 & Intermedio \\
\hline STP062 & 0.15117051 & Intermedio \\
\hline STP025 & 0.09758411 & Sensible \\
\hline ST162 & 0.09145114 & Sensible \\
\hline STI168 & 0.02853526 & Sensible \\
\hline
\end{tabular}

Los resultados encontrados permiten indicar la ausencia de poblaciones del patógeno resistente a Cimoxanil en las zonas productoras de papa en el Departamento de Nariño, indicando que la presión de selección no es muy fuerte y que el uso de fungicidas a base de Cimoxanil aún es un control eficiente de la enfermedad. Esto se debe principalmente a que la molécula ya no es utiliza de manera intensiva por la aparición de nuevas moléculas preventivas y curativas para el control de $P$. infestans, lo que facilita realizar rotaciones de ingredientes activos entre aplicaciones.

A pesar que Cimoxanil es calificado por la FRAC (2018) con un nivel de riesgo bajo, el hecho de encontrar aislamientos de $P$. infestans con valores de $\mathrm{EC}_{50}$ superiores hasta cinco veces con respecto al promedio encontrado en el resto de la población, es un indicativo de la necesidad de continuar con el monitoreo y especialmente de establecer medidas que eviten la aparición de aislamientos resistentes al producto, reduciendo la utilización de productos cuyo ingrediente activo sistémico sea Cimoxanil como un producto erradicante y alternando su aplicación con otros fungicidas sistémicos que presenten diferentes ingredientes activos y modos de acción (Gullino et al., 1997).

\section{Fluopicolide 6\% + Propineb 66,7\%}

La inhibición del crecimiento del patógeno se debe a que el fungicida interrumpe la división celular y la mitosis actuando sobre una proteína conocida como espectrina, la cual desempeña un papel vital en la estabilidad del citoesqueleto del patógeno, además induce lisis del micelio (Toquin et al., 2007; Briggs et al., 2006; FRAC, 2018). Por otra parte, la actividad antiesporulante se lleva a cabo primero con la detención, seguido del hinchamiento y ruptura de las estructuras reproductivas (esporangio y zoosporas) después de un minuto de ser tratados con el fungicida (Toquin et al., 2007).

Los resultados de esta investigación difieren a los obtenidos por Saville et al. (2015) reportan una EC-50 promedio de $P$. infestans frente a el ingrediente activo Fluopicolide $(99.1 \%)$ de $0.47 \mu \mathrm{g} \mathrm{mL}^{-1}$. Por otro lado, Keinath y Kousik (2011) en su evaluación de sensibilidad de aislamientos de P. infestans frente a Fluopicolide reportan valores de EC-50 valores promedio de 0,22 $\mu \mathrm{g} \mathrm{mL} \mathrm{mL}^{-1}$. Es importante destacar que los resultados de estos trabajos difieren con los reportados en esta investigación debido a que las metodologías de evaluación utilizadas y la fuente de la molécula química 
empleada fueron diferentes, pues en dichos trabajos se utilizó el ingrediente activo en grado técnico con una concentración del 99,1\%, mientras que en este estudio la fuente correspondió a una formulación comercial, en la que el ingrediente activo Fluopicolide se encuentra al $6 \%$ además de estar acompañado por el fungicida protectante Propineb al 66,7\%.

\section{Tabla 10. Clasificación de aislamientos de $P$. infestans según los valores de dosis media efectiva $\left(\mathrm{EC}_{50}\right)$ del fun- gicida Fluopicolide 6\% + Propineb 66,7\%.}

\begin{tabular}{|c|c|}
\hline Aislamiento & EC $_{50}$ \\
\hline STP067 & 7,429858586 \\
\hline STA157 & 7,289353415 \\
\hline ST1155 & 5,58909584 \\
\hline STG075 & 5,332464088 \\
\hline SPT056 & 5,009433128 \\
\hline STP046 & 4,787867895 \\
\hline STP115 & 3,995937667 \\
\hline STA083 & 3,124509027 \\
\hline STA078 & 2,502246318 \\
\hline STI006 & 1,41583901 \\
\hline STG100 & 1,15449664 \\
\hline STP025 & 1,037538963 \\
\hline ST1003 & 0,814346463 \\
\hline SPP031 & 0,653942765 \\
\hline STP041 & 0,618132027 \\
\hline STP039 & 0,542802924 \\
\hline STP062 & 0,521781635 \\
\hline ST1168 & 0,506779778 \\
\hline STA084 & 0,041677145 \\
\hline ST162 & 0,025894094 \\
\hline
\end{tabular}

Hasta la fecha no se conocen reportes de resistencia de poblaciones $P$. infestans a Fluopicolide (FRAC, 2018), por tal razón es importante implementar programas anti resistencia como lo propone la FRAC, quienes recomiendan el uso de mezclas de tanque o mezclas comerciales que incluyan fungicidas del tipo fenilamidas con productos protectantes multisitio, además de establecer una línea base del nivel de sensibilidad de P. infestans a Fluopicolide, de modo que cambios a futuro encaminados a generar resistencia en las poblaciones de este patógeno puedan ser comparados con la línea base de sensibilidad establecida y de esa manera tener más control sobre el uso de productos con este ingrediente activo (Keinath y Kousik, 2011). Es importante destacar que este estudio que establece las $\mathrm{EC}_{50}$ para este ingrediente activo (Fluopicolide) será útil a futuro como punto de referencia para investigaciones posteriores puesto que hasta el momento no se han realizado escalas para determinar la sensibilidad de P. infestans frente a este fungicida.

\section{CONCLUSIONES}

Los aislamientos de Phytophthora infestans evaluados presentaron diferentes niveles de sensibilidad al fungicida compuesto por Metalaxil + Mancozeb, encontrando asilamientos resistentes $(20 \%)$ y medianamente sensibles (80\%), debido al uso intensivo de fungicidas que contienen Metalaxil, lo cual ha llevado a que las poblaciones presenten o puedan llegar a presentar un proceso de adaptación a la molécula dando lugar a bajos porcentajes de inhibición y altos valores de $\mathrm{EC}_{50}$.

La inhibición del crecimiento micelial y la esporulación de $P$. infestans se vio reducida para los tres fungicidas a partir de la dosis $100 \mu \mathrm{g} \mathrm{mL}^{-1}$, la diferencia se encontró en los valores de $\mathrm{EC}_{50}$ en donde los fungicidas a base de Cimoxanil + Propineb y Fluopicolide + Propineb presentaron valores más bajos a los del fungicida a base de Metalaxil + Mancozeb, encontrando para Cimoxanil + Propineb aislamientos sensibles (15\%) y medianamente sensibles (85\%) y ningún aislamiento resistentes.

\section{AGRADECIMIENTOS}

Este estudio fue financiado por la Gobernación de Nariño dentro del Sistema General de Regalías en la modalidad de Joven Investigador dentro del programa: "Fortalecimiento de Capacidades Regionales en Investigación, Desarrollo Tecnológico e Innovación en el Departamento de Nariño", el cual se encontraba bajo la coordinación de la Fundación CEIBA y el Grupo de Investigación Genética y Evolución de Organismos Tropicales de la Universidad de Nariño.

Conflicto de intereses: el manuscrito fue preparado y revisado por los autores, quienes declaran no tener algún conflicto de interés que coloquen en riesgo la validez de los resultados aquí presentados. 


\section{REFERENCIAS BIBLIOGRÁFICAS}

Briggs, G., D. Mansfield, B. Moloney, S. Gary y T. Wegmann. 2006. The discovery and chemistry of fluopicolide. A new estándar for Oomycetes disease control. Pflanzenschutz Nachr. 59, 141-152.

DANE, Departamento Administrativo Nacional de Estadística de Colombia. 2018. Encuesta Nacional Agropecuaria (ENA). Bogotá.

Escudero, M. 2005. Secuencia parcial del gen citocromo b de Phytophthora infestans y su relación con la resistencia al fenamidone. Trabajo de grado. Facultad de Ciencias Agropecuarias, Universidad Nacional de Colombia, Medellín, Colombia.

Erwin, D.C. y O.K. Ribeiro. 1996. Phytophthora diseases worldwide. APS Press, St. Paul, MN.

FRAC, Fungicide Resistance Action Committe. 2018. FRAC code list: fungicides stored by mode of action. Bruselas, Bélgica.

García, H., M. Marín, S. Jaramillo y J.M. Cotes. 2008. Sensibilidad de aislamientos colombianos de Phytophthora infestans a cuatro fungicidas sistémicos. Agron. Colomb. 26(1), 47-57.

Granada, N., C. Naranjo, J. Castaño y R. Castro. 2002. Efecto de once extractos vegetales sobre el tizón tardío causado por Phytophthora infestans (Mont) De Bary en papa (Solanum phureja). Agronomía 10(1-2), 2-20.

Grunwald, N.J., A.K. Stturbaum, G. Romero, E. Garay, H. Loyoza y W.E. Fry. 2006. Selection for fungicide resistance within a growing season in field populations of Phytophthora infestans at center of origin. Phytopathology 96, 1397-1403. Doi: 10.1094/PHYTO-96-1397

Gullino, M.I., E. Mescalchin y M. Mezzalama. 1997. Sensitivity to cymoxanil in populations of Plasmopara viticola in northern Italy. Plant Pathol. 46, 726-736. Doi: 10.1046/j.1365-3059.1997.d01-68.x

Jaramillo, S. 2004. Monografía sobre Phytophthora infestans (Mont) de Bary. Universidad Nacional de Colombia, Medellín, Colombia.

Keinath, A.P. y C.S. Kousik. 2011. Sensitivity of isolates of Phytohthora capsici from the Eastern United States to Fluopicolide. Plant Dis. 95(11), 1414-1419. Doi: 10.1094/PDIS-03-11-0242

Lagos, L.E. 2002. Aislamiento y caracterización genética de las poblaciones de Phytophthora infestans en las zonas productoras de papa Solanum tuberosum en el departamento de Nariño. Tesis de maestría. Facultad de Ciencias, Universidad del Valle, Cali, Colombia.

Marín, M. y J. Mira. 1998. Caracterización de razas fisiológicas y tipo de apareamiento en aislamientos de Phytophthora infestans (Mont.) De Bary, en diferentes pisos térmicos y hospedantes en el departamento de Antioquia. Trabajo de grado. Facultad de Ciencias Agropecuarias, Universidad Nacional de Colombia, Medellín, Colombia.

Montes, G., H. Lozoya, G. Mora, S. Fernández y N. Grünwald. 2012. Rendimiento de papa en función de epidemia por tizón tardío (Phytophthora infestans Mont. de Bary). Rev. Fitotec. Mex. 35(1), 69-78.

Nuninger, C., C. Steden, y T. Staub. 1995. The contribution of Metalaxyl-based fungicide mixtures to potato late blight control Phytophthora infestans 150. pp. 122-129. En: Dowley, L.J., E. Bannon, L.R. Cooke, T. Keane y E. O'Sullivan (eds.). European Association for Potato Research-Pathology Section Conference. Boole Press, Dublin, Irlanda.

Ochoa, D. 2004. Determinación de la sensibilidad en los aislamientos colombianos de Phytophthora infestans hacia Cymoxanil, Dimetomorf y Clorotalonil. Trabajo de grado. Facultad de Ciencias, Pontificia Universidad Javeriana, Bogotá.

Pérez, W. y G. Forbes. 2008. Manual técnico del tizón tardío de la papa. Centro Internacional de la Papa, Lima.

Santana, F.M., C.B. Gómez, C. Rombaldi, V.J. Bianchi y A. Reis. 2013. Characterization of Phytophthora infestans populations of souther Brazil in 2004 and 2005. Phytoparasitica 41, 557-568. Doi: 10.1007/ s12600-013-0316-y

Saville, A., K. Graham, N.J. Grunwald, K. Myers, W.E. Fry y J.B. Ristaino. 2015. Fungicide sensitivity of U.S. genotypes of Phytophthora infestans to six oomycete-targeted compounds. Plant Dis. 99(5), 659-666. Doi: 10.1094/PDIS-05-14-0452-RE

Toquin, V., F. Barja, C. Sirven y R. Beffa. 2008. Fluopicolide, a new Anti-oomycetes fungicide with a new mode of action inducin perturbation of a spectrin-like protein. pp. 675-681. En: Kramer, W. y U. Schirmer (eds.). Modern crop protection compounds. Wiley-VCH Verlag, Weinheim, Alemania. Doi: 10.1002/9783527619580. ch19

Wang, W.O., Z.O. Ma, X.F. Zhang, W.J. Zhang y M. David. 2002. Resistance to fungicides, mating types and fitness of Phytophthora infestans. Acta Phytopathol. Sin. $32(3), 278-283$. 